Pub. Mat. UAB

No 21 Oct. 1980

Actes VII JMHL

TRANSPORTE DE FORMAS DE VOLUMEN EN UNA VARIEDAD DIFERENCIABEE REAL

Javier Lafuente López

Dpto. de Geometria y Topologia

Universidad Complutense de Madrid

By means of the concept of pseudo-diver gence operator, wich is defined in this pa I per, the weakest structure allowing a para liel displacemente of volume forms is esta bished on a difterentiable manifold. The study of this structure leads to some re-sults relative to the parallel displace--ment of volume forms associated to a linear comection on the manifold.

Notaciones y Definiciones Preliminares

in esto trabajo $M$ representra una variedad diferenciable paracompacta y conexa de dimensión finita $n, X(M)$ el algebra de lie de los campos diferenciables en $M$ y $F(M)$ el ágebra de las fun-ciones diferenciables en $M$ con valores reales.

Una dersidad $\theta$ en $M$ subordina un operador div $: X(M) \rightarrow F(M)$ que será denominado operador divergencia usual (O.D.U.). Si M es orientable, una forma de volumen $S$ en $M$ da lugar a una densidad denotada por $j \Omega$ l(véase [1]) y se escribirá div $v_{S}$ en lugar de div $\Omega$ Dicho operador viene definido por la fórmula: (div $\left.{ }_{\Omega} X\right) \Omega=L_{X} \Omega$, donde $L_{\chi}$ denota la derivada de Lie respecto al campo $X$. (véase [3]). 
Una sección $S$ en $L(M)$, fibrado de referencias en $M$, se - : identitica con una base local de campos $\left(e_{1}, \ldots, e_{n}\right)$ en el domi-nio abierto $u$ de definición de $s, y$ si $\left(\alpha, \ldots, \alpha^{n}\right)$ en su base . dual de 1 -formas, ${ }^{s} s_{S}=\alpha^{1} A \ldots A \alpha^{n}$ es una forma de volumen que da $1 \underline{u}$ gar a un O.D.U. en $U$ denotado por div ${ }_{S}$.

La traza $w$ de una conexión ineal res la traza de la ma$\operatorname{triz}\left(w_{j}\right)$ de proyeccionesverticales de $\Gamma$ en $L(M)$ (véase $[2]$ ).

Por altimo, si $\sigma$ es curva integral maximal de un spray $\mathfrak{X}$ en $M$, al conjunto imagen de o se denominará trayectoria del spray (vêase 4 ).

Definición 1

Un operador div: $x(M) \longrightarrow F(M)$ se 11 amará operador pseudo-divergencia (O.S.D.) si satisface las siguientes condiciones para $X, Y \in X(M), y$ $f \in F(M):$

1) $\operatorname{div}(X+Y)=\operatorname{div}(X)+\operatorname{div}(Y)$

2) $\operatorname{div}(f X)=\bar{L}_{X} f+\operatorname{fdiv} X$

Si además se satisface la condición

3) $\operatorname{div}\left(L_{X} Y\right)=L_{X}(\operatorname{div} Y)-L_{Y}(\operatorname{div} X)$, se denominarä operador divergencia (O.D.). Se demuestra facilmente que cualquier O,S.D. (respectivimente o.D.) puede obtenerse a partir de uno dado por adición de 1 -formas en $M$ (resp. I formas cerradas).

\section{leorema 1}

Un operador div: $Z(M) \longrightarrow F(M)$ es 0 . D. si y sólo si, para cada $p \in M$ existe un entorno aiburto y conexo $U$ de $p$, y una forma de volumen $\Omega$ en U unfvocamente determinada salvo constantes multiplicativas no nulas por la condición: (div $X) U=d i v ~(X / U)$ para todo $X \in X(M)$, o más bre mente, $\operatorname{div} / U=\operatorname{div}_{\Omega}$. 
J1 teorema 1 muestra que wn 0.0. es "localmente" un O.D.U.,y esto permite establecer respecto a él una definición localmente trivial de transporte de formas de volumen, que se extiende de forma natural para un $0.5 .1 .:$

\section{Definición (y Proposición) 2}

a) Sea div O.D. en $M, a:[a, b] \rightarrow M$ un camino continuo con origen $\rightarrow$ en $p \in M y \quad a_{p}$ forma de volumen en TM. Exite entonces una única forma de volumen continua a lo largo de o, $\Omega(t)=\left(0 \Omega_{p}\right) \mathrm{div}(\mathrm{t}) \mathrm{a} \leq \mathrm{t} \leq \mathrm{b}, \mathrm{ta} 1$ que:

i) $s_{i}(a)=s_{p}$

2) $\forall t_{0} \in[a, b\}$ existe $U$ entorno abierto de $\sigma\left(t_{0}\right), y$ forma: de volu--

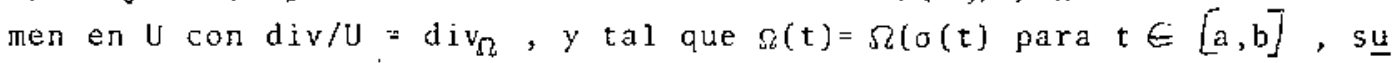
ficientemente próximo a $t_{\text {o }}$.

b) Pro otra parte, si div' $v^{\prime}$ div $+\alpha$ es 0.S.D. ( $\alpha$ es 1 -forma en $\left.M\right) y$ oes diferenciable a trozos, la Eórmula que sigue establece sin ambi-.. griedad una detiniciôn de transporte de formas de volumen respecto al operador div':

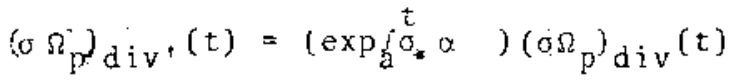

Un O.D. div. en $M$ determina de manera natural una $T$-forma en $L(M)$, w que queda bien definida por la condicion:

$$
W / L(U)=-\frac{d \Omega}{\Omega}-
$$

para cada forma de volumen $\Omega$ definida sobre un abierto $U$ de $M$, ta 1 que div/U $=$ div $\Omega$. esta iuea se generaliza para un O.S.D. en el siguiente

\section{Teorema 2}

Dado un O.S.D. div en $M$, existe una tinica 1 -forma w en $L(M)$ tal qu para toda sección local $5: \mathrm{J} \longrightarrow \mathrm{L}(\mathrm{M})$ es div/U $=\mathrm{di} v_{S}+\mathrm{S}^{*}$ w. Ademâs se verifican las equivalencias: 
1) div es 0.D. $\leftrightarrow$. es cerrada

2) div es O.D.U. $\Leftrightarrow w$ es exacta

Por otra parte sc demuestra que la traza $w$ de una colloxión lineal en $M$, es 1a 1 -forja en $L(M)$ asociada a un cierto O.S.D. div!', que define el mismo transporte de formas de volumen que la conexión. Si w es cerrada por el teorema 2 , div es 0.0 . y dicho transporte es localme te trivial.

Por otra parte para conexiones simetricas $\Gamma$, el carácter cerrado de w equivale a la simetría de su tensor de curvatura de Ricci, obteniendose el siguiente resultado:

\section{$\underline{\text { Teorema } 3}$}

Una conexión simetrica $\Gamma$ conserva localmente formas de volumen por transporte paralelo si y sólo si su tensor de curvatura de Ricci es si mêtrico.

Finalmente el teorema 4 tratará de precisar qué tipo de restriccic nes deben imponerse a una conexión lineal en M para que quede unívoca. mente determinada por su O.S.D. asociado:

\section{Teorema}

Sea $X$ un spray en M. Entonces para cada O.S. D div existe una unica conexiôn 1 ineal simétrica $F$ en $M$, con $\operatorname{div}_{\Gamma}=\operatorname{div}, y$ cuyo spray de geodésicas posee las mismas trayectorias que el spray $\Varangle$.

Su demostración está relacionada con las técnicas y resultados obtenidos en $[4]$. 
BIFL IOGRAF IA

(1) M. BERGER, B. GOSTIAUX

(2) NOEL J.HICKS

(3) R. ABRAHAM, J. MARSEN

(4) J . LAFUENTE
GEOMETRIE DIFFERENT IELLE

Libraire Armand Colin Paris, 1972

NOTES OF DIFFERENT IABLE GEOMETRY

Van Nostrand Reinholds Mathematical

Studes, 1971.

FOUNDATIONS OF MECHRIVICS

W. A. Benjamin Inc. 1967.

SOBRE LOS OPERADORES HESSIANOS ASOCIADOS A SPRAYS QUE POSEEN LAS MISMAS T'RAYECTORIAS.

PREPRINT . 